\title{
Microscopic structure of the hydrogen-phosphorus complex in crystalline silicon
}

\author{
P. J. H. Denteneer \\ Faculty of Science, Electronic Structure of Materials, Catholic University Nijmegen, \\ Toernooiveld 1, 6525 ED Nijmegen, The Netherlands \\ C. G. Van de Walle \\ Philips Laboratories, North American Philips Corporation, 345 Scarborough Road, Briarcliff Manor, New York 10510 \\ S. T. Pantelides \\ IBM Research Division, Thomas J. Watson Research Center, Yorktown Heights, New York 10598
}

(Received 8 August 1989)

\begin{abstract}
The existing discrepancy between theoretical models and experimental results for hydrogendonor complexes in crystalline silicon is resolved using first-principles pseudopotential-densityfunctional calculations for the hydrogen-phosphorus pair. In the configuration which is the global energy minimum, $\mathrm{H}$ is located on the extension of a $\mathrm{P}-\mathrm{Si}$ bond on the $\mathrm{Si}$ side, with the $\mathrm{Si}-\mathrm{H}$ pair relaxing away from $\mathrm{P}$ by $0.6 \AA$, leaving the $\mathrm{P}$ atom threefold coordinated. The calculated stretching and wagging vibrational frequencies associated with this configuration are in accord with experiment.
\end{abstract}

The structure and properties of hydrogen-impurity complexes in semiconductors have been studied intensively in the last few years using both experimental and theoretical methods. ${ }^{1-12}$ For the hydrogen-boron complex in silicon, which is the prototypal hydrogen-acceptor complex that has been studied most elaborately, a consistent picture has emerged (see, e.g., Ref. 5 and references therein). In the equilibrium configuration of the complex, the $\mathrm{H}$ atom resides inside a $\mathrm{Si}-\mathrm{B}$ bond, forming a three-center bond. Also for the qualitatively different $(\mathrm{H}, \mathrm{Be})$ pair in $\mathrm{Si}$ and $(\mathrm{H}, \mathrm{Si})$ pair in $\mathrm{Ge}$, theory has provided satisfactory explanations of the experimental results as well as new insights (see Ref. 6 and references therein). The $\mathrm{H}$ atom in the $(\mathrm{H}, \mathrm{Be})$ pair is able to tunnel around the $\mathrm{Be}$ atom because its lowest-energy location is close to the $C$ site (midway between two $\mathrm{Si}$ atoms bonded to $\mathrm{Be}$ ), where the relaxation of the surrounding $\mathrm{Si}$ atoms is small. The $\mathrm{H}$ atom in the $(\mathrm{H}, \mathrm{Si})$ complex in $\mathrm{Ge}$ is located close to a tetrahedral interstitial $\left(T_{d}\right)$ site.

In contrast, the structure of hydrogen-donor complexes, e.g., $(\mathrm{H}, \mathrm{P})$ in $\mathrm{Si}$, has so far not been determined conclusively. Experiments ${ }^{8}$ have shown that all $\mathrm{H}$-donor pairs in $\mathrm{Si}$ have similar infrared absorption spectra, suggesting that $\mathrm{H}$ is not bonded to the donor. The observation of a nondegenerate stretching mode around 1560 $\mathrm{cm}^{-1}$ and a doubly degenerate wagging mode around 810 $\mathrm{cm}^{-1}$ suggests that the center has trigonal symmetry. Theoretical models have so far not reproduced these frequencies. In Ref. 7, a model was proposed in which $\mathrm{H}$ is located on the extension of a P-Si bond on the side of $\mathrm{Si}$. Using empirical tight-binding calculations this "AB (antibonding) of Si" configuration was found to be lower in energy than the "AB of P" configuration. The frequency for the $\mathrm{H}$ stretching mode was calculated to be $2145 \mathrm{~cm}^{-1}$, which is very different from the experimentally determined value of $1555 \mathrm{~cm}^{-1.8}$ In a subsequent calculation by the same group, but using the more reliable firstprinciples pseudopotential-density-functional method, the configuration was qualitatively confirmed. ${ }^{10}$ However, in the latter calculation the stretching mode was found to be at $400 \mathrm{~cm}^{-1}$.

Recently, a number of groups using various kinds of cluster calculations ${ }^{4,11,12}$ have proposed a configuration similar to the one in Ref. 10 with the distinction, however, that the $\mathrm{Si}$ atom closest to $\mathrm{H}$ relaxes from its lattice site towards $\mathrm{H}$ to become almost coplanar with its three nearest-neighbor Si atoms. Estreicher et al. ${ }^{11}$ discuss the inherent difficulties in calculating vibrational frequencies to within a reasonable accuracy using quantum-mechanical cluster calculations and do not attempt to calculate any frequency. DeLeo and Fowler ${ }^{4}$ and Amore Bonapasta $e t$ al. ${ }^{12}$ calculate a $\mathrm{H}$ stretching frequency of 2150 $\mathrm{cm}^{-1}$, again in disagreement with experiment.

Summarizing, it can be said that theoretical studies so far have not been able to put forward a microscopic model for the $(H, P)$ complex that can be conclusively identified as the one that is experimentally observed.

In this paper, we present results of accurate firstprinciples calculations for the $(H, P)$ pair. We determine the lowest-energy configuration and show that this configuration is responsible for stretching and wagging vibrational frequencies that are in agreement with experiment. We have successfully used the pseudopotentialdensity-functional method before in studies of $\mathrm{H}$ in pure $\mathrm{Si}$ and of various complexes in $\mathrm{Si}$ and $\mathrm{Ge} .^{5,6,9}$ If the calculations are properly converged with respect to all the numerical approximations involved, the method is very reliable in determining defect configurations. In particular, total-energy differences between different defect configurations can be calculated to within an accuracy of 0.05-0.1 eV and typical $\mathrm{H}$ vibrational frequencies can be calculated with an accuracy of about $100 \mathrm{~cm}^{-1}$ (Ref. 5). For details of calculations in which such accuracy is achieved we refer to Refs. 5 and 9. In the present study, we closely examine various configurations with trigonal symmetry (see below), as well as the regions close to the $C$ 
and $C^{\prime}$ sites (i.e, $\mathrm{H}$ midway between two $\mathrm{Si}$ atoms bonded to $P$ and $H$ midway between $P$ and a next-nearestneighbor $\mathrm{Si}$, respectively). ${ }^{5}$ The $C$ and $C^{\prime}$ sites are at least $1.5 \mathrm{eV}$ higher in energy than the lowest-energy configuration; we will not consider them further. The configurations with trigonal symmetry, which can be classified according to the order in which the $\mathrm{H}, \mathrm{Si}$, and $\mathrm{P}$ atoms are found along a $\langle 111\rangle$ axis (H-Si-P, Si-H-P, and Si-P-H, respectively), are optimized by relaxing up to nine atoms according to the Hellmann-Feynman forces on these atoms. These forces can be calculated with the same level of accuracy as total energies from the self-consistent solutions of the Schrödinger equation for the valence electrons. ${ }^{13}$ In order to optimize the configurations we move the atoms in the direction of the calculated forces until the forces become negligible, thereby minimizing the total energy.

We find that each of the three trigonal-symmetry configurations, including appropriate relaxations of all the atoms, constitutes a local minimum of the total-energy surface. Furthermore, the three minima are very close in energy: they all lie in an energy range of only $0.5 \mathrm{eV}$ (see Fig. 1). These small energy differences open the way for the occurrence of metastable states of the complex.

Now we describe the two local minima and one global minimum configurations mentioned above. Of these three, the configuration highest in energy is the one in which $\mathrm{H}$ resides between a $\mathrm{Si}$ and $\mathrm{P}$ atom forming a bond. We call this configuration "BC (LLR of P)" since it involves a very large lattice relaxation (LLR) of the $P$ atom (BC stands for bond-center site). The $\mathrm{P}$ atom relaxes outward (away from $\mathrm{H}$ ) by $1.22 \AA$, whereas the $\mathrm{Si}$ atom relaxes outward by only $0.10 \AA$. The $\mathrm{H}$-Si distance in this configuration is $1.50 \AA$, similar to the $\mathrm{H}-\mathrm{Si}$ distances found in molecules, e.g., $\mathrm{SiH}_{4}$, and at a hydrogenated vacancy. The $\mathrm{H}$ atom breaks the $\mathrm{Si}-\mathrm{P}$ bond and saturates the Si dangling bond; this allows for the large relaxation of $\mathrm{P}$ through the plane of its three neighboring $\mathrm{Si}$ atoms to a position where it is threefold coordinated. The charge density for this configuration is shown in Fig. 2(a) and displays a lone pair on the $\mathrm{P}$ atom pointing in the direction of the nearest $T_{d}$ site on the line Si-H-P. The H-Si bond that is formed has a calculated stretch frequency of 1900 $\mathrm{cm}^{-1}$, much larger than the observed frequency. In the other local minimum configuration, which we call $A B$ of $\mathrm{P}$, the $\mathrm{H}$ atom is located very close to the $T_{d}$ site closest to the $\mathrm{P}$ atom. The energy of $\mathrm{AB}$ of $\mathrm{P}$ is only $0.10 \mathrm{eV}$ lower

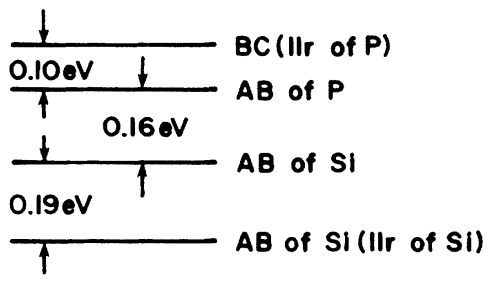

FIG. 1. Relative energies of different configurations with trigonal symmetry for $(\mathrm{H}, \mathrm{Si}, \mathrm{P})$ complexes in silicon. AB stands for antibonding site, $\mathrm{BC}$ for bond-center site, and LLR for large lattice relaxation. A more detailed description of the four configurations is given in the text (see also Fig. 3). than that of BC (LLR of P) (see Fig. 1). In this configuration, none of the atoms relax appreciably from their ideal lattice position, resulting in a H-P distance of almost an undistorted $\mathrm{Si}-\mathrm{Si}$ bond length $(2.35 \AA)$. The calculated $H$ stretch frequency for this configuration is $570 \mathrm{~cm}^{-1}$, much smaller than the observed frequency. Finally, the global energy minimum configuration is the one called $\mathrm{AB}$ of $\mathrm{Si}$ (LLR of $\mathrm{Si}$ ). It has an energy $0.35 \mathrm{eV}$
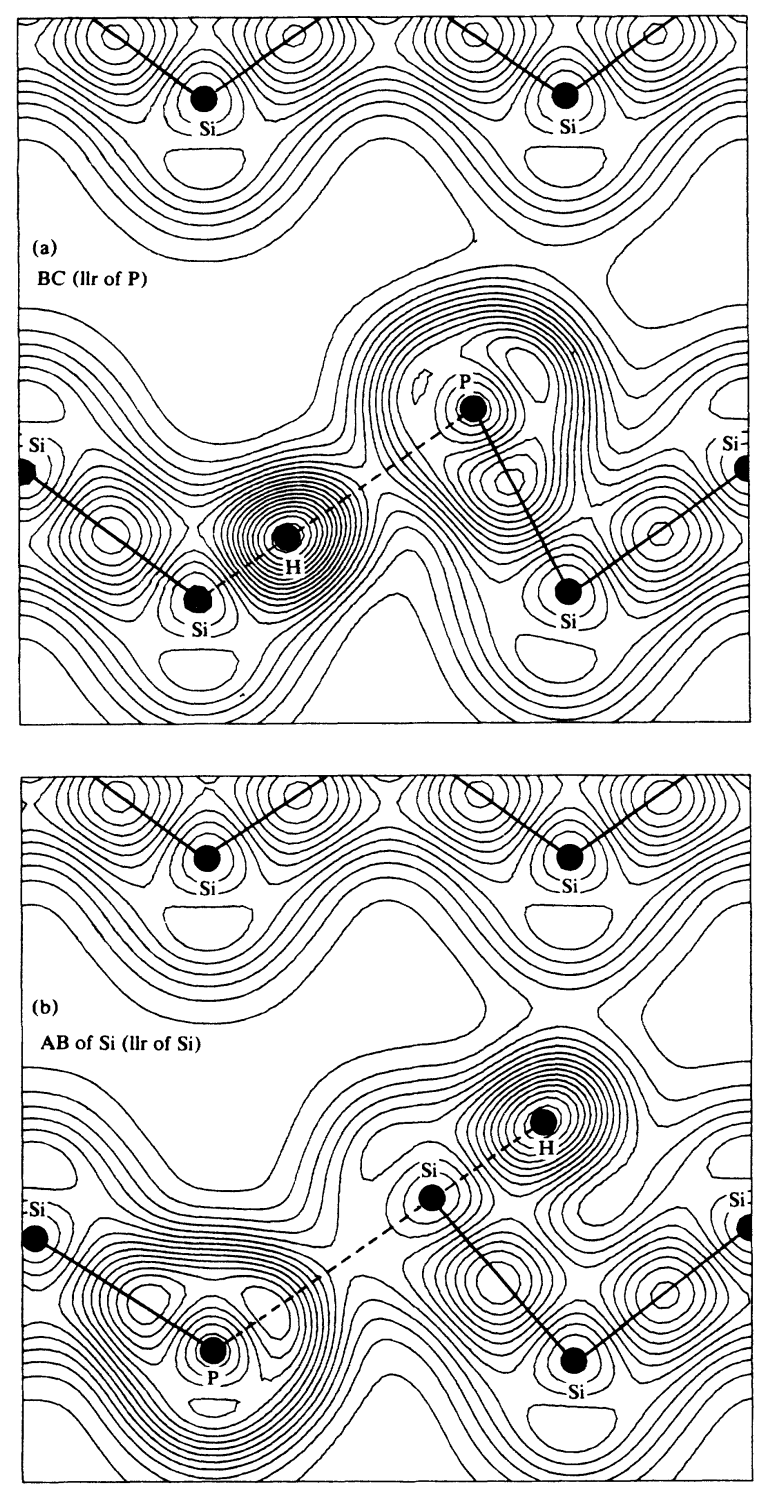

FIG. 2. Total valence charge density in the (110) plane for (a) the BC (LLR of P) and (b) the AB of Si (LLR of Si) configurations for a $(\mathrm{H}, \mathrm{Si}, \mathrm{P})$ complex in $\mathrm{Si}$. The black dots indicate atomic positions and the straight lines connect bonded atoms. The broken $\mathrm{Si}-\mathrm{P}$ bond and the $\mathrm{Si}-\mathrm{H}$ bond are indicated by dashed lines. The contour spacing is $1.87 \mathrm{e} / \Omega$, where $\Omega$ is the unit cell volume of bulk $\mathrm{Si}$ (which contains 8 electrons in bulk $\mathrm{Si}$ ). The lowest-density contour shown (in the channels between the two atomic chains) is $2.32 \mathrm{e} / \Omega$ and the highestdensity contour shown (around the $\mathrm{H}$ atomic position) is $\mathbf{3 4 . 1}$ $e / \Omega$ in (a) and $28.5 e / \Omega$ in (b). The maximum density in a $\mathrm{Si}-\mathrm{Si}$ bond is $24.0 \mathrm{e} / \Omega$. 
lower than $\mathrm{AB}$ of $\mathrm{P}$, and $\mathrm{H}$ is located close to the $T_{d}$ site of a $\mathrm{Si}$ atom bonded to $\mathrm{P}$. This $\mathrm{Si}$ atom relaxes outward by $0.59 \AA$ (leaving the $\mathrm{P}$ atom threefold coordinated; see Fig. 3). The $P$ atom relaxes by the small amount of 0.14 $\AA$ (in the direction of the Si relaxation, contrary to the results of cluster calculations). The $\mathrm{H}-\mathrm{Si}$ distance is $1.66 \AA$, which is somewhat larger than a typical value for a $\mathrm{H}-\mathrm{Si}$ bond distance (see above), indicating a slight weakening of the bond. The $\mathrm{H}$ stretch frequency is therefore expected to be lower than for a typical $\mathrm{Si}-\mathrm{H}$ bond. Indeed, we calculate a frequency of $1460 \mathrm{~cm}^{-1}$, which in view of the error bar on calculated frequencies discussed above, is in agreement with the experimental number of $1555 \mathrm{~cm}^{-1}$. Also the calculated frequency of the $\mathrm{H}$ wagging mode of $740 \mathrm{~cm}^{-1}$ is in agreement with the experimental result of $809 \mathrm{~cm}^{-1}$. The agreement of both calculated frequencies with experiment, taken together with the fact that the $A B$ of $\mathrm{Si}$ (LLR of $\mathrm{Si}$ ) configuration has the lowest energy of all configurations studied justifies the identification of the experimentally observed complex with this $\mathrm{AB}$ of $\mathrm{Si}$ (LLR of $\mathrm{Si}$ ) configuration.

In Fig. 2(b), we show the valence charge density of the $(\mathrm{H}, \mathrm{P})$ pair in the $\mathrm{AB}$ of $\mathrm{Si}$ (LLR of $\mathrm{Si}$ ) configuration. The P-Si bond is effectively broken and a lone-pair-like density, which is a remnant of the previous $\mathrm{P}-\mathrm{Si}$ bond, is extending in the direction of the former bond. All the valence electrons of $P$ are accounted for in this way. The $\mathrm{Si}$ atom has gone from an $s p^{3}$ bonding configuration to an $s p^{2}$ bonding configuration with respect to its three $\mathrm{Si}$ neighbors. The surplus electron of $\mathrm{Si}$ (which does not have to go in a $\mathrm{P}-\mathrm{Si}$ bond) pairs with the $\mathrm{H}$ electron to form a $\mathrm{Si}-\mathrm{H}$ bond. Indeed, the charge density between $\mathrm{Si}$ and $\mathrm{H}$ is very similar to the one found in the case of $\mathrm{H}$ saturating a Si dangling bond. Bonding is indicated by the fact that the charge density around the $\mathrm{H}$ atom is clearly modified from the spherical form it has when $\mathrm{Si}$ and $\mathrm{H}$ are far apart (see, e.g., the charge density for the AB of Si configuration in Ref. 10).

For the sake of completeness and to make the connection with the results of other work, we mention that if we do not allow for relaxation of the $\mathrm{Si}$ neighbors of the $\mathrm{Si}$ atom between $\mathrm{H}$ and $\mathrm{P}$, this $\mathrm{Si}$ atom relaxes outward by only $0.19 \AA$. This results in a $\mathrm{AB}$ of $\mathrm{Si}$ (without large lattice relaxation of $\mathrm{Si}$ ) configuration which is still lower in energy by $0.16 \mathrm{eV}$ than the $\mathrm{AB}$ of $\mathrm{P}$ configuration (see Fig. 1), but higher in energy by $0.19 \mathrm{eV}$ than the $\mathrm{AB}$ of $\mathrm{Si}$ (LLR of $\mathrm{Si}$ ) configuration. For this $\mathrm{AB}$ of $\mathrm{Si}$ configuration, which is similar to the one found in Ref. 10, the $\mathrm{H}$-Si distance is $2.1 \AA$, much larger than a typical $\mathrm{H}-\mathrm{Si}$ bond distance, and the corresponding $\mathrm{H}$ stretch frequency is calculated to be $600 \mathrm{~cm}^{-1}$. The $\mathrm{H}$ wagging mode for this configuration has a calculated frequency of $600 \mathrm{~cm}^{-1}$ as well, indicating the absence of $\mathrm{H}$ bonding. The configuration that we find to be lowest in energy is almost the same as the one found in Refs. 4, 11, and 12. In those calculations, the $\mathrm{Si}$ atom relaxes by an amount between

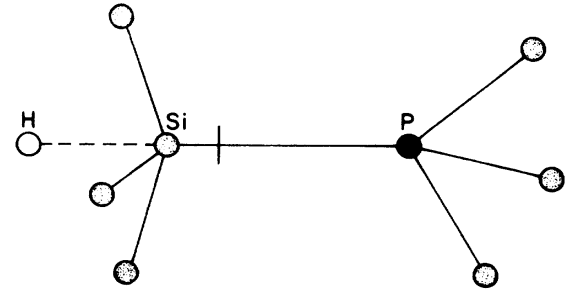

FIG. 3. Schematic representation of the AB of Si (LLR of $\mathrm{Si})$ configuration, which is the lowest-energy configuration for a $(\mathrm{H}, \mathrm{Si}, \mathrm{P})$ complex in $\mathrm{Si}$ (see also Fig. 1). One $\mathrm{Si}$ atom has relaxed from its lattice position (indicated by a vertical bar) by $0.59 \AA$ towards $\mathrm{H}$ and is only $0.19 \AA$ away from being coplanar with its three Si neighbors.

0.6 and $0.8 \AA$ and a Si-H distance between 1.4 and $1.5 \AA$ is found, which is smaller than our value of $1.66 \AA$. Consequently, those calculations render a much larger stretching mode frequency of about $2150 \mathrm{~cm}^{-1}$. More recently, Chadi et al. ${ }^{14}$ repeated the calculations of Ref. 10 and found similar results to those presented here by us.

Both configurations with large lattice relaxations discussed above are reminiscent of recently proposed models for the $E L 2$ and $D X$ defect centers in GaAs. ${ }^{15,16}$ In the case of $E L 2$, it is proposed that an As antisite can be induced by optical excitation to move by about $1.3 \AA$ from its lattice position to a metastable configuration. ${ }^{15}$ In the case of the $D X$ center, a Si donor in GaAs may move 1.2 $\AA$ from its lattice site. ${ }^{16}$ In both cases, the configuration with a large lattice relaxation is inherently associated with a simple point defect and can be provoked to materialize. In the subject of our present study, it is the $\mathrm{H}$ atom with its one unpaired electron that is able to promote different bonding environments for the simple substitutional $P$ donor involving large lattice relaxations of either a $\mathrm{P}$ or $\mathrm{Si}$ atom. In this way, the $\mathbf{P}$ atom can yield to its natural tendency to be threefold coordinated. The configuration with a large lattice relaxation of $\mathrm{Si}$ is found to be lowest in energy. We suggest that such complexes with large lattice relaxations be further investigated experimentally by means of ion-channeling techniques to confirm our findings.

In conclusion, we have shown on the basis of firstprinciples calculations of total energy that the configuration with $\mathrm{H}$ at an antibonding position of a $\mathrm{Si}$ neighbor of $\mathrm{P}$, in which this $\mathrm{Si}$ atom relaxes by $0.6 \AA$, can be identified with the complex that is experimentally observed. In doing so, the discrepancy between results of earlier theoretical studies and experiments is resolved.

This work was supported in part by the U.S. Office of Naval Research under Contract No. N00014-84-C-0396. One of the authors (P.J.H.D.) thanks the IBM Research Division for hospitality during part of the execution time of the work presented here. 
'S. J. Pearton, J. W. Corbett, and T. S. Shi, Appl. Phys. A 43, 153 (1987).

${ }^{2}$ E. E. Haller, in Proceedings of the Third International Conference on Shallow Impurities in Semiconductors, Linköping, 1988, edited by B. Monemar, IOP Conf. Ser. (Institute of Physics and The Physical Society, London, 1989), p. 425.

${ }^{3}$ M. Stavola, S. J. Pearton, J. Lopata, and W. C. DautremontSmith, Phys. Rev. B 37, 8313 (1988).

${ }^{4} G$. DeLeo and W. B. Fowler, in Hydrogen in Semiconductors, edited by J. I. Pankove and N. M. Johnson (unpublished); Bull. Am. Phys. Soc. 34, 834 (1989).

${ }^{5}$ P. J. H. Denteneer, C. G. Van de Walle, and S. T. Pantelides, Phys. Rev. B 39, 10809 (1989).

${ }^{6}$ P. J. H. Denteneer, C. G. Van de Walle, and S. T. Pantelides, Phys. Rev. Lett. 62, 1884 (1989).

${ }^{7}$ N. M. Johnson, C. Herring, and D. J. Chadi, Phys. Rev. Lett.
56, 769 (1986).

${ }^{8}$ K. Bergman, M. Stavola, S. J. Pearton, and J. Lopata, Phys. Rev. B 37, 2770 (1988).

${ }^{9}$ C. G. Van de Walle, P. J. H. Denteneer, Y. Bar-Yam, and S. T. Pantelides, Phys. Rev. B 39, 10791 (1989).

${ }^{10}$ K. J. Chang and D. J. Chadi, Phys. Rev. Lett. 60, 1422 (1988).

${ }^{11}$ S. K. Estreicher, L. Throckmorton, and D. S. Marynick, Phys. Rev. B 39, 13241 (1989).

${ }^{12}$ A. Amore Bonapasta, A. Lapiccirella, N. Tomassini, and M. Capizzi, Phys. Rev. B 39, 12630 (1989).

${ }^{13}$ M. T. Yin and M. L. Cohen, Phys. Rev. B 26, 3259 (1982).

${ }^{14}$ D. J. Chadi et al. (private communication).

${ }^{15} \mathrm{~J}$. Dabrowski and M. Scheffler, Phys. Rev. Lett. 60, 2183 (1988); D. J. Chadi and K. J. Chang, ibid. 60, 2187 (1988).

${ }^{16}$ D. J. Chadi and K. J. Chang, Phys. Rev. B 39, 10063 (1989). 